\title{
Review Article \\ Endothelial Progenitor Cells in Diabetic Microvascular Complications: Friends or Foes?
}

\author{
Cai-Guo Yu, ${ }^{1,2}$ Ning Zhang, ${ }^{1,2}$ Sha-Sha Yuan, ${ }^{1,2}$ Yan Ma, ${ }^{1,2}$ Long-Yan Yang, ${ }^{1,2}$ \\ Ying-Mei Feng, ${ }^{1,2}$ and Dong Zhao ${ }^{1,2}$ \\ ${ }^{1}$ Beijing Key Laboratory of Diabetes Prevention and Research, Luhe Hospital, Capital Medical University, Beijing 101149, China \\ ${ }^{2}$ Department of Endocrinology, Luhe Hospital, Capital Medical University, Beijing 101149, China
}

Correspondence should be addressed to Dong Zhao; zdoc66@126.com

Received 23 November 2015; Revised 5 January 2016; Accepted 18 April 2016

Academic Editor: Catherine M. Verfaillie

Copyright (C) 2016 Cai-Guo Yu et al. This is an open access article distributed under the Creative Commons Attribution License, which permits unrestricted use, distribution, and reproduction in any medium, provided the original work is properly cited.

Despite being featured as metabolic disorder, diabetic patients are largely affected by hyperglycemia-induced vascular abnormality. Accumulated evidence has confirmed the beneficial effect of endothelial progenitor cells (EPCs) in coronary heart disease. However, antivascular endothelial growth factor (anti-VEGF) treatment is the main therapy for diabetic retinopathy and nephropathy, indicating the uncertain role of EPCs in the pathogenesis of diabetic microvascular disease. In this review, we first illustrate how hyperglycemia induces metabolic and epigenetic changes in EPCs, which exerts deleterious impact on their number and function. We then discuss how abnormal angiogenesis develops in eyes and kidneys under diabetes condition, focusing on "VEGF uncoupling with nitric oxide" and "competitive angiopoietin 1/angiopoietin 2" mechanisms that are shared in both organs. Next, we dissect the nature of EPCs in diabetic microvascular complications. After we overview the current EPCs-related strategies, we point out new EPCs-associated options for future exploration. Ultimately, we hope that this review would uncover the mysterious nature of EPCs in diabetic microvascular disease for therapeutics.

\section{Introduction}

Prelude. Diabetes is a type of metabolic disorder, featured as insulin resistance and insufficient insulin release due to pancreatic $\beta$ cell dysfunction. Hyperglycemia appears in the early stage of diabetes. As the disease progresses, patients display excess thirst (polydipsia), frequent urination (polyuria), increased hunger (polyphagia), and loss of body weight. As most pathological changes involved in blood vessels of multiple organs, macro- and microvascular complications are frequently observed in diabetic patients and become the major cause of mortality.

Endothelial progenitor cells (EPCs) were first described nearly two decades ago. They participate in endothelial repair either by secreting angiogenic factors or by incorporating into disrupted endothelium and differentiating into endothelial cells to maintain endothelium integrity. Despite the longterm debate on the nature and identification of EPCs, compelling data showed that EPCs improved blood perfusion in peripheral ischemia. Nevertheless, abnormal angiogenesis is the featured pathological hallmark in diabetic retinopathy and nephropathy and, therefore, anti-VEGF treatment has been applied for treating the microvascular abnormality. Thus, the questions are rising: what is the nature of EPCs in diabetic microvascular disease? Could we apply EPCs for the treatment of diabetic retinopathy and nephropathy?

The Presence of EPCs in Nature. EPCs were first described in 1997. When $\mathrm{CD}_{3} 4^{+}$cells were isolated from human peripheral blood, they could differentiate into endothelial cells in vitro and participate in angiogenesis in vivo [1]. Despite a long debate about EPC identity, more and more data collectively indicated the presence of EPCs in nature: (1) human induced pluripotent cells (hiPSCs) could differentiate into vascular endothelial progenitors that could incorporate into injured endothelium in vivo $[2,3]$; (2) despite being putative, both adult and human embryonic stem cells-derived hemangioblasts have shown endothelial capacities [4]; (3) 
different mechanical cues could sense cardiosphere-derived cells with enriched $\mathrm{cKit}^{+}$subpopulation to differentiate either to endothelial or to cardiomyogenic lineage [5]; (4) Prox $-1^{+}$ cells emerging at E9.5 could sprout from the veins to form lymph sacs and an initial lymphatic vasculature [6].

Heterogeneous EPCs Population. Data from different groups consistently demonstrated that EPCs are heterogeneous populations and classified into early EPCs and late EPCs. Early EPCs, also known as colony-forming unit-ECs (CFU-ECs) or CFU-Hill, exhibited a spindle shaped morphology, had poor proliferative capacity, and produced to a high extent angiogenic cytokines $[1,7]$. By contrast, late EPCs, now generally termed as endothelial colony-forming cells (ECFCs), showed a cobblestone-shaped morphology and highly proliferative activity when cultured in vitro. In response to injury, they could be mobilized from bone marrow or other locations, migrate toward lesion site, and incorporate into injured endothelium in vivo [8-12]. Therefore, ECFCs are the main target under investigation.

Identification and Cultivation of EPCs. So far, no unique surface marker has been identified for EPCs. Instead, different combinations of surface markers have been used for EPCs identification, such as CD $34^{+} / \mathrm{VEGFR}^{+}$and $\mathrm{CD} 133^{+} / \mathrm{VE}-$ cadherin $^{+}$[13-16]. Although these markers could help us to quantify EPCs in vivo, it is yet uncertain whether the cells isolated from these markers could give rise to ECFCs in vitro. Thus, functional assays become more reliable for EPCs confirmation, which include their morphology, ability to form vasculature in vitro, and incorporation into vasculature upon injection in vivo.

With regard to cultivation, early EPCs could be obtained from mononuclear cells (MNCs) of human peripheral blood or cord blood after 4-5 days of culture in medium containing endothelial growth factors and fetal calf serum [7, 17]. They are recognized by monocytic morphology, uptake of acetylated low-density lipoprotein (AcLDL), and binding to lectin.

To obtain ECFCs, mononuclear cells are cultured and passaged in endothelial cell specific medium with endothelial growth factors for at least 28 days. During culture, endothelial colonies appear and can be picked up for further expansion [16]. As the development of stem cell technology, embryonic stem cells and induced pluripotent stem cells (iPSCs) have become useful alternative cell sources to generate EPCs for practice $[2,3,18,19]$.

Reduced Number and Impaired Function of EPCs in Diabetes. Besides their huge capacity for endothelium generation and maintenance of endothelium integrity, a recent observation indicated the beneficial effect of EPCs on $\beta$ cell survival. When pancreatic $\beta$ cells were cotransplanted with EPCs, a better $\beta$ cell engraftment with preserved function was observed, resulting in improved cure rate and initial glycemic control [20]. Unfortunately, EPCs number was significantly reduced with impaired function in diabetic patients as well as $\mathrm{db} / \mathrm{db}$ mice, which was associated with poor vascular outcome in diabetes $[21,22]$. In the next section, we discuss how hyperglycemia induces metabolic and epigenetic changes in EPCs.

\section{Metabolic and Epigenetic Change of EPCs in Diabetes}

2.1. Hyperglycemia-Associated Metabolic Change. Hyperglycemia induces advanced glycated end products (AGEs) formation and oxidative stress and increases reactive oxygen species (ROS) production in mitochondrion, which are the main killers of EPCs apoptosis [23]. Increased ROS production could also stimulate AGEs production, which further triggers ROS production. To make it worse, both of them synergistically activate nuclear factor-kappa B (NF$\kappa \mathrm{B})$ transcription [24]. NF- $\kappa \mathrm{B}$ is a well-known transcription factor with most of its target genes encoding inflammatory proteins inducing interleukin $1 \beta$ (IL-1 $\beta$ ) and tumor necrosis factor- $\alpha$ (TNF- $\alpha)$ and p53 as well as inducible nitric oxide synthase (iNOS). Thus, a loop between iNOS, ROS, NF- $\kappa$ B, and AGEs develops to aggravate inflammation cascade [2527]. Apart from ROS production in mitochondrion, high concentration of glucose provokes endoplasmic reticulum stress (ER stress), which promotes EPCs apoptosis and reduces EPCs migratory function [28]. Autophagy is a homeostatic process and is involved in organelle recycling and protein degradation. However, in response to high glucose-induced ER stress and oxidative stress, excessive autophagy might contribute to EPCs death [29].

In line with increased apoptosis by hyperglycemia, high level of glucose jeopardizes EPCs proliferative capacity partially via its inhibition of Akt phosphorylation and subsequently NOS activation or via activation of C-Jun N-terminal kinase (JNK) pathway [30-32]. High glucose also induces EPCs senescence by one of NF- $\kappa \mathrm{B}$ target genes, p53, and activation of p38MAPK pathway [33]. Figure 1 summarizes the molecular mechanisms of how hyperglycemia adversely affected EPCs number and function.

2.2. Hyperglycemia-Associated Epigenetic Change. Glycemic control is the initial treatment for diabetes. Nonetheless, tight glycemic control in the late stage of the disease fails to attenuate diabetic vascular complications. This phenomenon of glycemic memory was noticed in 1987 [34] and was extensively validated by different diabetic animal models and preclinical findings. These reports consistently pointed out that intensive glycemic control in the early stage could delay the development of diabetic microvascular abnormalities in type-1 [35] and type-2 diabetic subjects $[36,37]$ as well as streptozotocin-induced diabetic rats [38].

Glycemic memory is defined as the inexorable progression of diabetic vascular complication that is linked to uncontrolled hyperglycemia in the early stage of diabetes despite a tight glycemic control in the follow-on period. How does glycemic memory come? In vitro data have demonstrated that exposure of aortic endothelial cells to high glucose for 16 hours promotes NF- $\kappa \mathrm{B}$ p 65 gene transcription. This transcription activation is sustained even when endothelial cells are cultivated back to normal glucose concentration [39]. Similarly, when Zheng et al. challenged retinal endothelial cells with high glucose conditions for one week and then returned to normal glucose conditions for two weeks, they noticed that one week of hyperglycemia was sufficient to 


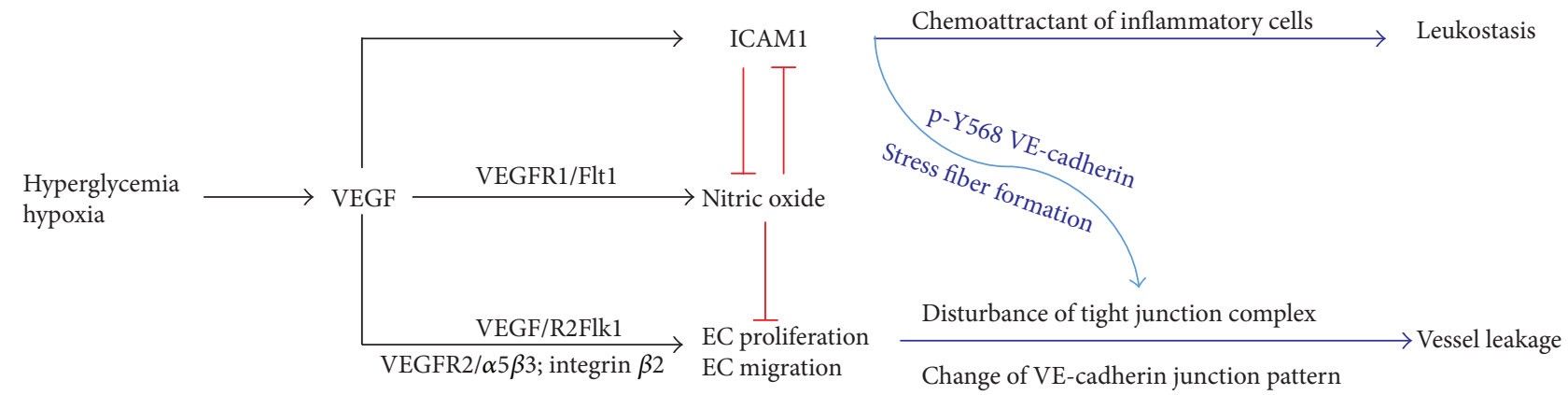

FIGURE 1: Uncoupling VEGF with nitric oxide in microvascular endothelial cells. Hyperglycemia promoted local VEGF production. VEGF stimulated endothelial cell proliferation via binding to VEGFR2. It induced endothelial cell migration and altered junction complex, in particular, VE-cadherin on the membrane. In parallel, VEGF promoted ICAM1 expression in endothelial cells which triggered inflammatory response to attack endothelium and made VE-cadherin dissemble. ICAM1 could also enhance ROS production that has negative impact on nitric oxide level and bioavailability. Although VEGF promoted nitric oxide production in endothelial cells via its receptor Flt1, nitric oxide is significantly reduced by ROS and oxidative stress, losing its inhibitory effect on VEGF-induced endothelial cell proliferation, migration, and activation.

induce NF- $\kappa$ B activation which remained unchanged for the remaining two weeks [40]. They further found that Sirtuin 1 (SIRT1) was the main regulator in the event. Belonging to a class 3 HDAC, SIRT1 deacetylates H3K14 and H14K16 to control ROS production in endothelial cells and positively regulates EPCs differentiation into endothelial lineage [41].

Epigenetic modulation mainly includes posttranslational histone modification, DNA methylation, and microRNAregulated transcriptional changes. As mentioned above, hyperglycemia-induced oxidative stress, ROS, and AGEs are the main factors for EPCs apoptosis and dysfunction. They are also potent inducers for epigenetic changes in EPCs. For instance, ROS is associated with a series of histone modifications in the promoter and enhancer of superoxide dismutase 2 (SOD2) gene in rat retinal endothelial cells [42]. High glucose triggers the increase of $\mathrm{H} 3 \mathrm{~K} 4 \mathrm{mel}$ but decreases $\mathrm{H} 3 \mathrm{~K} 9 \mathrm{me} 2$ and $\mathrm{H} 3 \mathrm{~K} 9 \mathrm{me} 3$ expression level at the promoter of NF- $\kappa \mathrm{B}$ in human microvascular endothelial cells, leading to NF- $\kappa \mathrm{B}$ activation [43]. Furthermore, the histone codes H3K9ac, H3K12ac, H3K4me2, and H3K4me3 suppress eNOS transcription, leading to decreased nitric oxide [44]. All these epigenetic modifications accelerate proinflammatory machinery for EPCs apoptosis and loss of function.

It is well accepted that EPCs-mediated endothelium repair is beneficial for coronary heart disease, whereas abnormal angiogenesis is the hallmark of diabetic retinopathy and nephropathy and anti-VEGF agents have been applied for treating diabetic microvascular diseases. Thus, the question is coming: how do EPCs participate in the microvascular disease? Next, we first review how abnormal angiogenesis initiates diabetic microvascular disease.

\section{Abnormal Angiogenesis in Diabetic Retinopathy and Nephropathy}

Diabetic retinopathy is one of most frequent complications in diabetes, which is the leading cause of vision loss. After 20 years of diabetes, almost all type- 1 diabetes patients, $80 \%$ of insulin-dependent diabetics, and 50\% of insulin-independent type-2 diabetic patients will develop retinopathy [45, 46]. Diabetic retinopathy is traditionally classified into two main clinical forms: nonproliferative diabetic retinopathy (NPDR) and proliferative diabetic retinopathy (PDR), based on the presence or absence of neovascularization.

In a similar situation to diabetic nephropathy, 20\%$40 \%$ of diabetic patients develop nephropathy [47], which is featured as initial microalbuminuria and then followed by a lot of albuminuria and increased serum creatinine level. Diabetic nephropathy has become the leading cause of end stage renal disease worldwide.

From the pathological view of diabetic retinopathy and nephropathy, aberrant angiogenesis is the common feature in the diseases, which is characterized as hypoxia-induced local VEGF expression, reducing nitric oxide level and availability, oxidative stress, vascular leakage, and inflammation. In parallel with VEGF axis, the imbalanced expression between angiotensin I and angiotensin II serves as another mechanism for endothelial dysfunction and hyperpermeability in diabetic eyes and kidneys.

3.1. Uncoupling VEGF with Nitric Oxide. Endothelium acts as physiological barrier between serum proteins and blood vessel, whose integrity is tightly controlled by nitric oxide produced by endothelial cells. Physical level of VEGF promotes endothelial cell proliferation via its receptor VEGFR2 and stimulates eNOS activation for nitric oxide (NO) production via VEGFR1.

In the setting of diabetes, hypoxia-induced VEGF production acts on endothelial cells for proliferation, migration, and NO production; in the meantime, it stimulates intercellular cell adhesion molecule-1 (ICAM1) expression on the surface of endothelial cells $[48,49]$. ICAM1 triggers NADPH oxidase activation for reactive oxygen species (ROS) production [48]. ROS, advanced glycation end products (AGEs), asymmetric dimethylarginine (ADMA), and hyperglycemia dramatically increase arginase activity [50]. Arginase competes with NOS for the common substrate, L-arginine, resulting in insufficient substrate for NOS. Uncoupled NOS in turn 


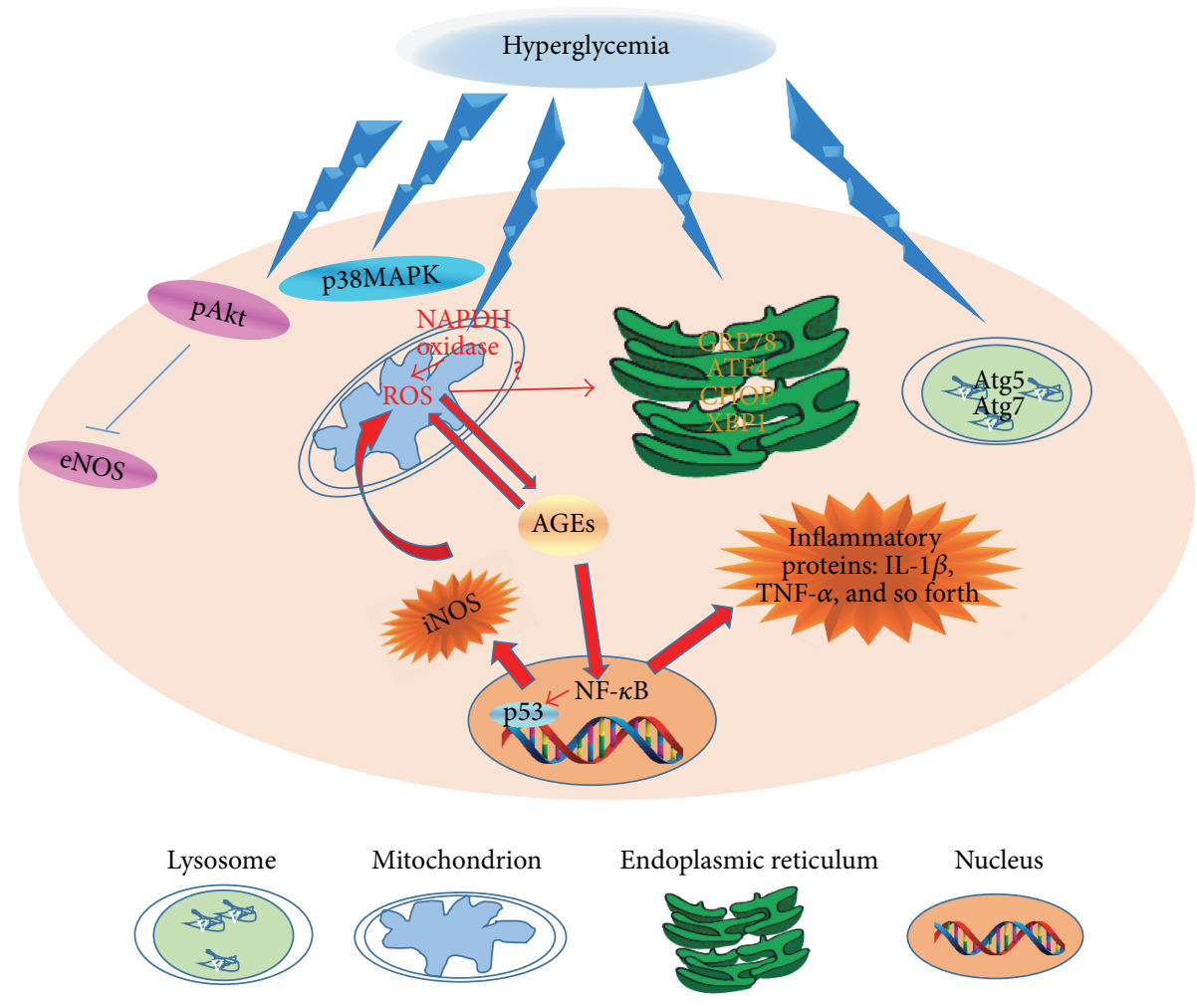

Figure 2: The detrimental effect of hyperglycemia on endothelial progenitor cells (EPCs) number and function. High level of glucose stimulates ROS production through activation of NAPDH oxidase. Increased reactive oxygen species (ROS) production triggers advanced glycated end products (AGEs) formation. AGE in turn further increases ROS production and, in the meantime, promotes nuclear factor-kappa $\mathrm{B}(\mathrm{NF}-\kappa \mathrm{B})$ transcription. NF- $\kappa \mathrm{B}$ is crucially involved in inflammation via transcriptional activation of its target genes such as IL-1 $\beta$ and TNF$\alpha$. In parallel, NF- $\kappa \mathrm{B}$ also activates $\mathrm{p} 53$ to accelerate cell senescence and iNOS that further potentiates ROS production. Except mitochondrial diabetic retinopathy ion damage, hyperglycemia induces endoplasmic reticulum (ER) stress and excessive autophagy to further facilitate EPCs death. Apart from that, hyperglycemia inhibits Akt phosphorylation and subsequently eNOS activation. It also activates p38MAPK pathway to promote cell death and senescence. Ultimately, EPCs are triggered to undergo apoptosis and become dysfunctional.

use more oxygen molecules to generate superoxide, which catabolizes any available NO for peroxynitrite formation [50]. In addition, ADMA is an endogenous inhibitor of eNOS but its level is increased in diabetes [51]. Ultimately, NO level and availability are severely reduced.

In contrast to diminished $\mathrm{NO}$ availability, VEGF induces abnormal endothelial cell proliferation via VEGFR2 and, more importantly, enhances migration but disrupts cytoskeleton rearrangement through cross talk between VEGFR 2 and integrin $\alpha 5 \beta 3$ or integrin $\beta 2$, separately $[52,53]$. Moreover, under physical angiogenesis, Notch/VEGFR2 modulates differential dynamics of VE-cadherin junction pattern during sprouting. When switched to pathological high VEGF condition, the differential VE-cadherin mobility is lost and thus tip cell competition and stalk cell intercalation are disturbed [54]. In parallel, downstream of VEGF-induced ICAM1 expression and ROS production, Src kinase and protein tyrosine kinase $2 \beta$ are activated, both of which phosphorylate Y-658 on VE-cadherin for dissembling this protein [55]. Moreover, ICAM1 could also activate Rho GTPase for stress fiber formation, leading to permeability [55]. Nitric oxide antagonizes endothelial cell proliferation and inflammation, thereby maintaining endothelium integrity [56].
The pathological pattern "VEGF uncoupling with NO" is preserved and serves as the main mechanism in diabetic retinopathy and nephrology $[52,57]$. For instance, studies from diabetic eNOS knockout mice have demonstrated that this mouse model develops severe albuminuria as well as increased VEGF expression in the kidney. Histological analysis confirms diabetic nephropathy in this model as evidenced by mesangial expansion, glomerular basement membrane thickening, and mesangiolysis [57]. Concerning the eye, VEGF level has been found to be significantly increased in ocular tissues in diabetic patients with retinopathy, which is accompanied with inflammation and uncoupled eNOS $[58,59]$.

Taken together, irregular endothelial proliferation, migration, cytoskeleton rearrangement, and dissembled VE-cadherin contribute to vessel leakage. The "uncoupling of VEGF with NO" pattern is shown in Figure 2.

3.2. Angiopoietins in Diabetic Eye and Kidney. On top of "VEGF uncoupling with NO," angiopoietin 1 (Ang1)/Tie2 is another system that protects endothelium integrity. Upon binding to Tie2 tyrosine kinase receptor, Ang1 has been 
shown to reduce endothelium permeability, suppress NF- $\kappa \mathrm{B}-$ associated inflammation, and antagonize VEGF functions. In contrast, Ang2 is the endogenous antagonist of Ang1. The increase of Ang2 in diabetes condition competes with Ang1 for binding to Tie2, rendering the Ang1-regulated antiangiogenesis toward Ang2-mediated abnormal angiogenesis [60]. Recent study reported that VEGF and Angl exert opposing effect on endothelial cell permeability via their distinct modulation of RhoA-specific guanine nucleotide exchange factor (Syx). In the study, the authors elaborately showed that Syx was recruited to endothelial junction by Muppl and formed a complex with multiple members of the apicobasal polarity complexes (CRB) on the membrane for junction stabilization. They further demonstrated that VEGF caused Syx dissociation from Muppl and Syx translocation from cell junctions, resulting in junction disassembly [61].

A potent Angl variant, cartilage oligomeric matrix protein (COMP), was developed nearly one decade ago. Administration of COMP-Ang1 reverses hyperglycemia-induced kidney dysfunction by suppression of ICAM1 and monocyte chemoattractant protein-1 and monocyte/macrophage infiltration in diabetic $\mathrm{db} / \mathrm{db}$ mice [60]. COMP-Ang1 also reduces renal tissue levels of transforming growth factor-betal (TGF$\beta 1$, alpha-smooth muscle actin, and fibronectin, as well as Smad 2/3 expression, but increases Smad 7 expression [60]. Likewise, recent data demonstrate that COMP-Ang1 could ameliorate retinopathy and stabilize blood retinal barrier in diabetic Ins2Akita mice [63].

\section{EPCs in Diabetic Retinopathy and Nephropathy}

4.1. EPCs in Diabetic Retinopathy. After observing the presence of abnormal angiogenesis in the development of diabetic retinopathy, antiangiogenesis therapies such as antiVEGF agents have been taken to treat diabetic patients with retinopathy. This has brought a long debate: are EPCs good or bad in the disease? There is no definite answer so far. As the complicated pathogenesis and different types of diabetic retinopathy, either decreased or increased or unchanged EPCs number has been reported in diabetic patients with severe retinopathy when comparing to diabetic patients with no to mild retinopathy or healthy subjects [64-68].

To be noted, in the studies where they found increased EPCs number in the patients with diabetic retinopathy, EPCs function such as migration and mobilization and homing was often impaired. And this EPCs pattern, that is, increased EPCs number with impaired function, is consistently conserved in both type- 1 and type- 2 diabetic patients $[68,69]$. Paradoxically, intravitreal delivery of COMP-Ang1 improves endothelial integrity and ameliorates vascular leakage by promoting the incorporation of endothelial colony-forming cells into retinal vasculature [63] in diabetic mice. Giving the nature of ECFCs in endothelial regeneration and $\mathrm{NO}$ production, this is an excellent example illustrating using the right ECFCs in reversing diabetic retinopathy.
4.2. EPCs in Diabetic Nephropathy. The early pathological features of diabetic nephropathy include hyperperfusion and hyperfiltration due to endothelial cell damage and abnormal angiogenesis. As the inflammation becomes more severe, glomeruli fibrosis develops, resulting in kidney failure. Although the exact mechanisms of nephropathy are not fully understood, AGEs, oxidative stress, and the activation of the renin-angiotensin-aldosterone system (RAAS) facilitate and strengthen these changes partially through activation of TGF$\beta 1$ signaling and increased vascular endothelial growth factor (VEGF) expression in the kidney toward progression of fibrosis and renal failure [70-72]. Therefore, antagonism of VEGF signaling using anti-VEGF antibody or endogenous inhibitor of VEGF or inhibition of VEGF receptor-1 phosphorylation has been used to improve early renal function in diabetic rats injected with STZ or $\mathrm{db} / \mathrm{db}$ mice [73-75]. In parallel, inhibition of AGEs suppresses TGF- $\beta 1$ and VEGF signaling pathways and alleviated diabetic nephropathy [72].

As endothelial cell damage occurs in the early stage of kidney dysfunction, its repair is not well processed due to EPCs defect in diabetes. In line with this, Makino and coworkers reported the negative correlation between EPCs number and microalbuminuria or albumin excretion rate in both type- 1 and type- 2 diabetic patients, respectively $[76,77]$, suggesting the protective effect of EPCs in the structure and function of glomeruli.

Putting these evidences together, ECFCs could be a promising target for treating diabetic retinopathy and nephropathy. Giving that ECFCs number and function are reduced in diabetic condition, how to obtain sufficient amount of ECFCs with desirable function for therapeutic is under investigation.

\section{ECFC Therapy for Diabetic Microvascular Disease}

5.1. Drugs. Till present, some of the antidiabetic drugs like metformin, thiazolidinediones, GLP-1 agonists, DPP-4 inhibitors, and insulin might increase EPCs number and improve EPCs function with increase of nitric oxide bioavailability [78-87]. Except for these antidiabetic drugs, lipidlowering drugs, statins, improve EPCs number and function [88]. Table 1 provides an overview of these antidiabetic drugs in the aspects of their effects on EPCs number and function.

5.2. Cord- and Cord Blood-Derived ECFCs. For clinical practice, ECFCs can be obtained from long-term cultivation of mononuclear cells isolated from blood. Nevertheless, the quality and functionality of ECFCs could vary from one group to another, which could be due to the different passages and ECFCs purity they use and risk factors that the donors carry on [89].

Alternatively, induced pluripotent stem cells (iPSCs) generated from $\mathrm{CD} 34^{+}$cord blood cells have shown huge capability of differentiating into ECFCs. For therapeutic purposes, autologous iPSCs are more favored to avoid immune rejection. However, the experimental practice of iPSC generated from patients is always frustrating by growth arrest, 
TABLE 1: Overview of the effect of antidiabetic drugs on EPCs number and function.

\begin{tabular}{|c|c|c|c|}
\hline Author & Drug & Groups & Conclusion \\
\hline Sorrentino et al., 2007 & $\begin{array}{l}\text { Thiazolidinedione } \\
\text { (TZD) }\end{array}$ & $\begin{array}{l}\text { T2DM patients } \\
\text { T2DM: } n=30 \\
\text { Controls: } n=10\end{array}$ & $\begin{array}{l}\text { Rosiglitazone restored nitric oxide } \\
\text { bioavailability and improved EPC } \\
\text { function }\end{array}$ \\
\hline Spigoni et al., 2012 & $\begin{array}{l}\text { Thiazolidinedione } \\
\text { (TZD) }\end{array}$ & $\begin{array}{l}\text { In vitro cultured ECFC } \\
\text { isolated from subjects with } \\
\text { impaired glucose tolerance: } \\
n=14\end{array}$ & $\begin{array}{l}\text { Pioglitazone improved ECFC viability } \\
\text { and capacity to form tubular-like } \\
\text { structures }\end{array}$ \\
\hline Humpert et al., 2008 & Insulin & $\begin{array}{l}\text { ECFC culture, } \\
\text { in vitro }\end{array}$ & Insulin improved EPC function \\
\hline Fadini et al., 2011 & Insulin & $\begin{array}{l}\text { T2DM patients: } \\
n=42\end{array}$ & Insulin increased EPC count \\
\hline Maiorino et al., 2016 & Insulin & $\begin{array}{l}\text { T1DM patients } \\
\text { insulin infusion: } n=41\end{array}$ & $\begin{array}{l}\text { Insulin infusion increased EPC } \\
\text { number in T1DM patients }\end{array}$ \\
\hline Liao et al., 2010 & Metformin & $\begin{array}{l}\text { Newly diagnosed } \\
\text { T2DM: } n=46 \\
\text { Non-DM: } n=51\end{array}$ & $\begin{array}{l}\text { Metformin increased EPC number in } \\
\text { the blood }\end{array}$ \\
\hline Liu et al., 2011 [62] & GLP-1 agonists & $\begin{array}{l}\text { EPC culture, } \\
\text { in vitro }\end{array}$ & $\begin{array}{l}\text { GLP-1 enhanced EPC proliferation and } \\
\text { VEGF production in EPC }\end{array}$ \\
\hline Gonçalves et al., 2012 & DPP-4 inhibitors & Diabetic rats & $\begin{array}{l}\text { Sitagliptin increased the number of } \\
\text { CD } 34^{+} \text {cells in the blood }\end{array}$ \\
\hline Mohler III et al., 2009 & $\begin{array}{l}\text { Atorvastatin; } \\
\text { ezetimibe }\end{array}$ & Diabetic swines & $\begin{array}{l}\text { EPC number in the circulation was } \\
\text { increased by atorvastatin }\end{array}$ \\
\hline Chang et al., 2010 & Adiponectin & $\begin{array}{l}\text { Adiponectin deficient } \\
\mathrm{db} / \mathrm{db} \text { mice }\end{array}$ & Adiponectin rescued EPC senescence \\
\hline
\end{tabular}

uncontrolled differentiation, and incomplete function [90, 91]. Recent studies shed light on getting desired ECFCs. After iPSCs were obtained from healthy donors and patients with type-1 diabetes, the differentiation of iPSCs toward vascular cells was processed in an adherent, feeder-free differentiation protocol and further assembled in 3D engineered hyaluronic acid hydrogels for maturation. When injecting, the yielded endothelial progenitors were incorporated into vasculature [92]. In line with this study, Park et al. reported that vascular progenitors generated from human iPSCs derived from cord blood possessed greater capacity for homing and longterm incorporation into injured retinal vessels [2]. These studies establish promising strategies for applying iPSCderived endothelial progenitors for stabilizing microvascular structure and inhibiting vessel leakage.

5.3. Genetically Modified ECFC. When dissecting the two main pathological machineries that affect retinopathy and nephropathy, applying ECFC with higher level of nitric oxide or Ang1 would be favorable for stabilized capillaries by reversing "uncoupled VEGF with nitric oxide," balancing "Ang1/Ang2 competition" and "rendering Ang1/VEGF." This idea awaits future evaluation.

\section{Additional Points}

Endothelial progenitors play a major role in the maintenance of endothelium integrity. Unfortunately, hyperglycemia induces metabolic and epigenetic changes in EPCs, leading to EPC apoptosis and reduced function. Reduced
EPCs number and function jeopardize endothelial regeneration, resulting in hyperpermeability and inflammation in capillaries and then development of retinopathy and nephropathy. Providing the angiogenesis potential of incorporating into injured vessel and increased nitric oxide production upon stimulation, ECFCs might become promising and complimentary therapeutic targets for treating diabetic patients. iPSC-based ECFCs would be one of the main strategies for clinical therapeutic for diabetic microvascular abnormality. To gain intact endothelium and protect vessel from retinopathy and nephropathy, it might be advisable to administer ECFC in the early stage of diabetes for better efficacy.

\section{Competing Interests}

The authors declare no competing interests regarding the publication of this paper.

\section{Authors' Contributions}

Cai-Guo Yu, Ning Zhang, Sha-Sha Yuan, and Yan Ma contributed equally to the paper.

\section{Acknowledgments}

The study was financially funded by Tong-Zhou Science and Technology Committee in Beijing (no. KJ2015CX006). 


\section{References}

[1] T. Asahara, T. Murohara, A. Sullivan et al., "Isolation of putative progenitor endothelial cells for angiogenesis," Science, vol. 275, no. 5302, pp. 964-967, 1997.

[2] T. S. Park, I. Bhutto, L. Zimmerlin et al., "Vascular progenitors from cord blood-derived induced pluripotent stem cells possess augmented capacity for regenerating ischemic retinal vasculature," Circulation, vol. 129, no. 3, pp. 359-372, 2014.

[3] A. Peters, P. W. Burridge, M. V. Pryzhkova et al., "Challenges and strategies for generating therapeutic patient-specific hemangioblasts and hematopoietic stem cells from human pluripotent stem cells," The International Journal of Developmental Biology, vol. 54, no. 6-7, pp. 965-990, 2010.

[4] S.-J. Lu, Q. Feng, S. Caballero et al., "Generation of functional hemangioblasts from human embryonic stem cells," Nature Methods, vol. 4, no. 6, pp. 501-509, 2007.

[5] Kshitiz, J. Afzal, D.-H. Kim, and A. Levchenko, "Concise review: mechanotransduction via p190RhoGAP regulates a switch between cardiomyogenic and endothelial lineages in adult cardiac progenitors," Stem Cells, vol. 32, no. 8, pp. 19992007, 2014.

[6] J. Kazenwadel and N. L. Harvey, "Morphogenesis of the lymphatic vasculature: a focus on new progenitors and cellular mechanisms important for constructing lymphatic vessels," Developmental Dynamics, vol. 245, no. 3, pp. 209-219, 2016.

[7] J. M. Hill, G. Zalos, J. P. J. Halcox et al., "Circulating endothelial progenitor cells, vascular function, and cardiovascular risk," The New England Journal of Medicine, vol. 348, no. 7, pp. 593-600, 2003.

[8] J. Hur, C.-H. Yoon, H.-S. Kim et al., "Characterization of two types of endothelial progenitor cells and their different contributions to neovasculogenesis," Arteriosclerosis, Thrombosis, and Vascular Biology, vol. 24, no. 2, pp. 288-293, 2004.

[9] D. A. Ingram, L. E. Mead, H. Tanaka et al., "Identification of a novel hierarchy of endothelial progenitor cells using human peripheral and umbilical cord blood," Blood, vol. 104, no. 9, pp. 2752-2760, 2004.

[10] D. N. Prater, J. Case, D. A. Ingram, and M. C. Yoder, "Working hypothesis to redefine endothelial progenitor cells," Leukemia, vol. 21, no. 6, pp. 1141-1149, 2007.

[11] F. Timmermans, J. Plum, M. C. Yöder, D. A. Ingram, B. Vandekerckhove, and J. Case, "Endothelial progenitor cells: identity defined?" Journal of Cellular and Molecular Medicine, vol. 13, no. 1, pp. 87-102, 2009.

[12] M. C. Yoder, L. E. Mead, D. Prater et al., "Redefining endothelial progenitor cells via clonal analysis and hematopoietic stem/progenitor cell principals," Blood, vol. 109, no. 5, pp. 18011809, 2007.

[13] A. Avogaro, M. Albiero, L. Menegazzo, S. de Kreutzenberg, and G. P. Fadini, "Endothelial dysfunction in diabetes: the role of reparatory mechanisms," Diabetes Care, vol. 34, supplement 2, pp. S285-S290, 2011.

[14] M. L. Caramori, P. Fioretto, and M. Mauer, "The need for early predictors of diabetic nephropathy risk: is albumin excretion rate sufficient?" Diabetes, vol. 49, no. 9, pp. 1399-1408, 2000.

[15] M. L. Caramori, P. Fioretto, and M. Mauer, "Enhancing the predictive value of urinary albumin for diabetic nephropathy," Journal of the American Society of Nephrology, vol. 17, no. 2, pp. 339-352, 2006.
[16] M. C. Yoder, "Human endothelial progenitor cells," Cold Spring Harbor Perspectives in Medicine, vol. 2, no. 7, Article ID a006692, 2012.

[17] H. Ito, I. I. Rovira, M. L. Bloom et al., "Endothelial progenitor cells as putative targets for angiostatin," Cancer Research, vol. 59, no. 23, pp. 5875-5877, 1999.

[18] P. Campagnolo, T.-N. Tsai, X. Hong et al., "c-Kit+ progenitors generate vascular cells for tissue-engineered grafts through modulation of the Wnt/Klf4 pathway," Biomaterials, vol. 60, pp. 53-61, 2015.

[19] S. Rafii, C. C. Kloss, J. M. Butler et al., "Human ESC-derived hemogenic endothelial cells undergo distinct waves of endothelial to hematopoietic transition," Blood, vol. 121, no. 5, pp. 770780, 2013.

[20] S. Kang, H. S. Park, A. Jo et al., "Endothelial progenitor cell cotransplantation enhances islet engraftment by rapid revascularization," Diabetes, vol. 61, no. 4, pp. 866-876, 2012.

[21] M. Rigato, C. Bittante, M. Albiero, A. Avogaro, and G. P. A. Fadini, "Circulating progenitor cell count predicts microvascular outcomes in type 2 diabetic patients," The Journal of Clinical Endocrinology and Metabolism, vol. 100, no. 7, pp. 2666-2672, 2015.

[22] D. Singla and J. Wang, "Fibroblast growth factor-9 activates cKit progenitor cells and enhances angiogenesis in the infarcted diabetic heart," Oxidative Medicine and Cellular Longevity, vol. 2016, Article ID 5810908, 12 pages, 2016.

[23] N. Kränkel, V. Adams, A. Linke et al., "Hyperglycemia reduces survival and impairs function of circulating blood-derived progenitor cells," Arteriosclerosis, Thrombosis, and Vascular Biology, vol. 25, no. 4, pp. 698-703, 2005.

[24] Y. Zhang, X.-Y. Luo, D.-H. Wu, and Y. Xu, "ROR nuclear receptors: structures, related diseases, and drug discovery," Acta Pharmacologica Sinica, vol. 36, no. 1, pp. 71-87, 2015.

[25] J. Park, J.-S. Min, B. Kim et al., "Mitochondrial ROS govern the LPS-induced pro-inflammatory response in microglia cells by regulating MAPK and NF- $\kappa \mathrm{B}$ pathways," Neuroscience Letters, vol. 584, pp. 191-196, 2015.

[26] H.-L. Yang, P.-J. Huang, Y.-R. Liu et al., “Toona sinensis inhibits LPS-induced inflammation and migration in vascular smooth muscle cells via suppression of reactive oxygen species and NF- $\kappa \mathrm{B}$ signaling pathway," Oxidative Medicine and Cellular Longevity, vol. 2014, Article ID 901315, 16 pages, 2014.

[27] J. Zhen, H. Lu, X. Q. Wang, N. D. Vaziri, and X. J. Zhou, "Upregulation of endothelial and inducible nitric oxide synthase expression by reactive oxygen species," American Journal of Hypertension, vol. 21, no. 1, pp. 28-34, 2008.

[28] M. Bhatta, J. H. Ma, J. J. Wang, J. Sakowski, and S. X. Zhang, "Enhanced endoplasmic reticulum stress in bone marrow angiogenic progenitor cells in a mouse model of long-term experimental type 2 diabetes," Diabetologia, vol. 58, no. 9, pp. 2181-2190, 2015.

[29] K.-A. Kim, Y.-J. Shin, M. Akram et al., "High glucose condition induces autophagy in endothelial progenitor cells contributing to angiogenic impairment," Biological and Pharmaceutical Bulletin, vol. 37, no. 7, pp. 1248-1252, 2014.

[30] C. B. D. Sollier, N. Berge, B. Boval, L. Hovsepian, and L. Drouet, "Functional variability of platelet response to clopidogrel correlates with $\mathrm{P} 2 \mathrm{Y} 12$ receptor occupancy," Thrombosis and Haemostasis, vol. 101, no. 1, pp. 116-122, 2009.

[31] K. A. Gallagher, Z.-J. Liu, M. Xiao et al., "Diabetic impairments in NO-mediated endothelial progenitor cell mobilization and 
homing are reversed by hyperoxia and SDF- $1 \alpha$," The Journal of Clinical Investigation, vol. 117, no. 5, pp. 1249-1259, 2007.

[32] M. Ye, D. Li, J. Yang et al., "MicroRNA-130a targets MAP3K12 to modulate diabetic endothelial progenitor cell function," Cellular Physiology and Biochemistry, vol. 36, no. 2, pp. 712-726, 2015.

[33] A. Rosso, A. Balsamo, R. Gambino et al., "p53 mediates the accelerated onset of senescence of endothelial progenitor cells in diabetes," Journal of Biological Chemistry, vol. 281, no. 7, pp. 4339-4347, 2006.

[34] R. L. Engerman and T. S. Kern, "Progression of incipient diabetic retinopathy during good glycemic control," Diabetes, vol. 36, no. 7, pp. 808-812, 1987.

[35] J. M. Lachin, N. H. White, D. P. Hainsworth, W. Sun, P. A. Cleary, and D. M. Nathan, "Effect of intensive Diabetes therapy on the progression of diabetic retinopathy in patients with type 1 diabetes: 18 years of follow-up in the DCCT/EDIC," Diabetes, vol. 64, no. 2, pp. 631-642, 2015.

[36] R. R. Holman, S. K. Paul, M. A. Bethel, H. A. Neil, and D. R. Matthews, "Long-term follow-up after tight control of blood pressure in type 2 diabetes," The New England Journal of Medicine, vol. 359, no. 15, pp. 1565-1576, 2008.

[37] N. Azad, L. Agrawal, N. V. Emanuele, R. Klein, G. D. Bahn, and P. Reaven, "Association of blood glucose control and pancreatic reserve with diabetic retinopathy in the Veterans Affairs Diabetes Trial (VADT)," Diabetologia, vol. 57, no. 6, pp. 1124-1131, 2014.

[38] R. A. Kowluru, "Effect of reinstitution of good glycemic control on retinal oxidative stress and nitrative stress in diabetic rats," Diabetes, vol. 52, no. 3, pp. 818-823, 2003.

[39] A. El-Osta, D. Brasacchio, D. Yao et al., "Transient high glucose causes persistent epigenetic changes and altered gene expression during subsequent normoglycemia," The Journal of Experimental Medicine, vol. 205, no. 10, pp. 2409-2417, 2008.

[40] Z. Zheng, H. Chen, J. Li et al., "Sirtuin 1-mediated cellular metabolic memory of high glucose via the LKB1/AMPK/ROS pathway and therapeutic effects of metformin," Diabetes, vol. 61, no. 1, pp. 217-228, 2012.

[41] N. D’Onofrio, M. Vitiello, R. Casale, L. Servillo, A. Giovane, and M. L. Balestrieri, "Sirtuins in vascular diseases: emerging roles and therapeutic potential," Biochimica et Biophysica ActaMolecular Basis of Disease, vol. 1852, no. 7, pp. 1311-1322, 2015.

[42] Q. Zhong and R. A. Kowluru, "Epigenetic modification of Sod2 in the development of diabetic retinopathy and in the metabolic memory: role of histone methylation," Investigative Ophthalmology and Visual Science, vol. 54, no. 1, pp. 244-250, 2013.

[43] Y.-D. Li, B.-Q. Ye, S.-X. Zheng et al., "NF- $\kappa$ B transcription factor p50 critically regulates tissue factor in deep vein thrombosis," The Journal of Biological Chemistry, vol. 284, no. 7, pp. 44734483, 2009.

[44] J. E. Fish, C. C. Matouk, A. Rachlis et al., "The expression of endothelial nitric-oxide synthase is controlled by a cell-specific histone code," Journal of Biological Chemistry, vol. 280, no. 26, pp. 24824-24838, 2005.

[45] R. Klein, B. E. K. Klein, and S. E. Moss, "The Wisconsin Epidemiological Study of Diabetic Retinopathy: a review," Diabetes/Metabolism Reviews, vol. 5, no. 7, pp. 559-570, 1989.

[46] P. Romero-Aroca, R. Sagarra-Alamo, J. Basora-Gallisa, T. Basora-Gallisa, M. Baget-Bernaldiz, and A. Bautista-Perez, "Prospective comparison of two methods of screening for diabetic retinopathy by nonmydriatic fundus camera," Clinical Ophthalmology, vol. 4, no. 1, pp. 1481-1488, 2010.
[47] R. Gupta and A. Misra, "Epidemiology of microvascularcomplications of diabetes in south Asians and comparison with other ethnicities," Journal of Diabetes, 2016.

[48] M. Rojas, W. Zhang, Z. Xu et al., "Requirement of NOX2 expression in both retina and bone marrow for diabetesinduced retinal vascular injury," PLoS ONE, vol. 8, no. 12, article e84357, 2013.

[49] M. Al-Shabrawey, M. Rojas, T. Sanders et al., "Role of NADPH oxidase in retinal vascular inflammation," Investigative Ophthalmology and Visual Science, vol. 49, no. 7, pp. 3239-3244, 2008.

[50] S. P. Narayanan, M. Rojas, J. Suwanpradid, H. A. Toque, R. W. Caldwell, and R. B. Caldwell, "Arginase in retinopathy," Progress in Retinal and Eye Research, vol. 36, pp. 260-280, 2013.

[51] H. Cheng and R. C. Harris, "Renal endothelial dysfunction in diabetic nephropathy," Cardiovascular and Hematological Disorders-Drug Targets, vol. 14, no. 1, pp. 22-33, 2014.

[52] D. H. Jo, J. Bae, S. Chae et al., "Quantitative proteomics reveals $\beta 2$ integrin-mediated cytoskeletal rearrangement in vascular endothelial growth factor (VEGF)-induced retinal vascular hyperpermeability," Molecular \& Cellular Proteomics, vol. 15, no. 5, pp. 1681-1691, 2016.

[53] J. Wu, T. L. Strawn, M. Luo et al., "Plasminogen activator inhibitor-1 inhibits angiogenic signaling by uncoupling vascular endothelial growth factor receptor- $2-\alpha \mathrm{V} \beta 3$ integrin cross talk," Arteriosclerosis, Thrombosis, and Vascular Biology, vol. 35, no. 1, pp. 111-120, 2015.

[54] K. Bentley, C. A. Franco, A. Philippides et al., "The role of differential VE-cadherin dynamics in cell rearrangement during angiogenesis," Nature Cell Biology, vol. 16, no. 4, pp. 309$321,2014$.

[55] I. H. Sarelius and A. J. Glading, "Control of vascular permeability by adhesion molecules," Tissue Barriers, vol. 3, no. 1-2, article e985954, 2015.

[56] T. Nakagawa, T. Kosugi, M. Haneda, C. J. Rivard, and D. A. Long, "Abnormal angiogenesis in diabetic nephropathy," Diabetes, vol. 58, no. 7, pp. 1471-1478, 2009.

[57] T. Nakagawa, W. Sato, O. Glushakova et al., "Diabetic endothelial nitric oxide synthase knockout mice develop advanced diabetic nephropathy," Journal of the American Society of Nephrology, vol. 18, no. 2, pp. 539-550, 2007.

[58] E. Ulker, W. H. Parker, A. Raj, Z. Qu, and J. M. May, "Ascorbic acid prevents VEGF-induced increases in endothelial barrier permeability," Molecular and Cellular Biochemistry, vol. 412, no. 1-2, pp. 73-79, 2016.

[59] G. Tremolada, C. Del Turco, R. Lattanzio et al., "The role of angiogenesis in the development of proliferative diabetic retinopathy: impact of intravitreal anti-VEGF treatment," Experimental Diabetes Research, vol. 2012, Article ID 728325, 8 pages, 2012.

[60] S. Lee, W. Kim, S.-O. Moon et al., "Renoprotective effect of COMP-angiopoietin-1 in db/db mice with type 2 diabetes," Nephrology Dialysis Transplantation, vol. 22, no. 2, pp. 396-408, 2007.

[61] S. P. Ngok, R. Geyer, M. Liu et al., "VEGF and angiopoietin-1 exert opposing effects on cell junctions by regulating the Rho GEF Syx," Journal of Cell Biology, vol. 199, no. 7, pp. 1103-1115, 2012.

[62] W. Liu, H. Jin, K. A. Lee, S. Xie, H. S. Baek, and T. S. Park, "Neuroprotective effect of the glucagon-like peptide-1 receptor agonist, synthetic exendin-4, in streptozotocin-induced diabetic rats," British Journal of Pharmacology, vol. 164, no. 5, pp. 1410-1420, 2011. 
[63] J. M. Cahoon, R. R. Rai, L. S. Carroll et al., "Intravitreal AAV2.COMP-ang1 prevents neurovascular degeneration in a murine model of diabetic retinopathy," Diabetes, vol. 64, no. 12, pp. 4247-4259, 2015.

[64] J. V. Busik, M. Tikhonenko, A. Bhatwadekar et al., "Diabetic retinopathy is associated with bone marrow neuropathy and a depressed peripheral clock," The Journal of Experimental Medicine, vol. 206, no. 13, pp. 2897-2906, 2009.

[65] N. G. de la Torre, R. Fernández-Durango, R. Gómez et al., "Expression of angiogenic MicroRNAs in endothelial progenitor cells from type 1 diabetic patients with and without diabetic retinopathy," Investigative Ophthalmology and Visual Science, vol. 56, no. 6, pp. 4090-4098, 2015.

[66] S. Hazra, Y. P. R. Jarajapu, V. Stepps et al., "Long-term type 1 diabetes influences haematopoietic stem cells by reducing vascular repair potential and increasing inflammatory monocyte generation in a murine model," Diabetologia, vol. 56, no. 3, pp. 644-653, 2013.

[67] P. Hu, J. S. Thinschmidt, Y. Yan et al., "CNS inflammation and bone marrow neuropathy in type 1 diabetes," American Journal of Pathology, vol. 183, no. 5, pp. 1608-1620, 2013.

[68] K. Tan, E. Lessieur, A. Cutler et al., "Impaired function of circulating CD34(+) CD45(-) cells in patients with proliferative diabetic retinopathy," Experimental Eye Research, vol. 91, no. 2, pp. 229-237, 2010.

[69] G. Zerbini, A. Maestroni, A. Palini et al., "Endothelial progenitor cells carrying monocyte markers are selectively abnormal in type 1 diabetic patients with early retinopathy," Diabetes, vol. 61, no. 4, pp. 908-914, 2012.

[70] M. Furukawa, T. Gohda, M. Tanimoto, and Y. Tomino, "Pathogenesis and novel treatment from the mouse model of type 2 diabetic nephropathy," The Scientific World Journal, vol. 2013, Article ID 928197, 8 pages, 2013.

[71] Y. Kanesaki, D. Suzuki, G. Uehara et al., "Vascular endothelial growth factor gene expression is correlated with glomerular neovascularization in human diabetic nephropathy," American Journal of Kidney Diseases, vol. 45, no. 2, pp. 288-294, 2005.

[72] K. Tsuchida, Z. Makita, S. Yamagishi et al., "Suppression of transforming growth factor beta and vascular endothelial growth factor in diabetic nephropathy in rats by a novel advanced glycation end product inhibitor, OPB-9195," Diabetologia, vol. 42, no. 5, pp. 579-588, 1999.

[73] A. S. De Vriese, R. G. Tilton, M. Elger, C. C. Stephan, W. Kriz, and N. H. Lameire, "Antibodies against vascular endothelial growth factor improve early renal dysfunction in experimental diabetes," Journal of the American Society of Nephrology, vol. 12, no. 5, pp. 993-1000, 2001.

[74] T. Kosugi, T. Nakayama, Q. Li et al., "Soluble Flt-1 gene therapy ameliorates albuminuria but accelerates tubulointerstitial injury in diabetic mice," American Journal of Physiology - Renal Physiology, vol. 298, no. 3, pp. F609-F616, 2010.

[75] S. H. Sung, F. N. Ziyadeh, A. Wang, P. E. Pyagay, Y. S. Kanwar, and S. Chen, "Blockade of vascular endothelial growth factor signaling ameliorates diabetic albuminuria in mice," Journal of the American Society of Nephrology, vol. 17, no. 11, pp. 3093-3104, 2006.

[76] F. H. Bahlmann, K. De Groot, J.-M. Spandau et al., "Erythropoietin regulates endothelial progenitor cells," Blood, vol. 103, no. 3, pp. 921-926, 2004.

[77] H. Makino, S. Okada, A. Nagumo et al., "Decreased circulating $\mathrm{CD} 34+$ cells are associated with progression of diabetic nephropathy: short report," Diabetic Medicine, vol. 26, no. 2, pp. 171-173, 2009.

[78] J. Chang, Y. Li, Y. Huang et al., "Adiponectin prevents diabetic premature senescence of endothelial progenitor cells and promotes endothelial repair by suppressing the p38 MAP kinase/p16INK4A signaling pathway," Diabetes, vol. 59, no. 11, pp. 2949-2959, 2010.

[79] G. P. Fadini, S. V. de Kreutzenberg, V. Mariano et al., "Optimized glycaemic control achieved with add-on basal insulin therapy improves indexes of endothelial damage and regeneration in type 2 diabetic patients with macroangiopathy: a randomized crossover trial comparing detemir versus glargine," Diabetes, Obesity and Metabolism, vol. 13, no. 8, pp. 718-725, 2011.

[80] A. Gonçalves, E. Leal, A. Paiva et al., "Protective effects of the dipeptidyl peptidase IV inhibitor sitagliptin in the blood-retinal barrier in a type 2 diabetes animal model," Diabetes, Obesity and Metabolism, vol. 14, no. 5, pp. 454-463, 2012.

[81] P. M. Humpert, Z. Djuric, U. Zeuge et al., "Insulin stimulates the clonogenic potential of angiogenic endothelial progenitor cells by IGF-1 receptor-dependent signaling," Molecular Medicine, vol. 14, no. 5-6, pp. 301-308, 2008.

[82] Y.-F. Liao, L.-L. Chen, T.-S. Zeng et al., "Number of circulating endothelial progenitor cells as a marker of vascular endothelial function for type 2 diabetes," Vascular Medicine, vol. 15, no. 4, pp. 279-285, 2010.

[83] M. I. Maiorino, O. Casciano, E. D. Volpe, G. Bellastella, D. Giugliano, and K. Esposito, "Reducing glucose variability with continuous subcutaneous insulin infusion increases endothelial progenitor cells in type 1 diabetes: an observational study," Endocrine, vol. 52, no. 2, pp. 244-252, 2016.

[84] E. R. Mohler III, Y. Shi, J. Moore et al., "Diabetes reduces bone marrow and circulating porcine endothelial progenitor cells, an effect ameliorated by atorvastatin and independent of cholesterol," Cytometry Part A, vol. 75, no. 1, pp. 75-82, 2009.

[85] S. A. Sorrentino, F. H. Bahlmann, C. Besler et al., "Oxidant stress impairs in vivo reendothelialization capacity of endothelial progenitor cells from patients with type 2 diabetes mellitus: restoration by the peroxisome proliferator-activated receptor$\gamma$ agonist rosiglitazone," Circulation, vol. 116, no. 2, pp. 163-173, 2007.

[86] V. Spigoni, A. Picconi, M. Cito et al., "Pioglitazone improves in vitro viability and function of endothelial progenitor cells from individuals with impaired glucose tolerance," PLoS ONE, vol. 7, no. 11, Article ID e48283, 2012.

[87] X. Xiao-Yun, M. Zhao-Hui, C. Ke, H. Hong-Hui, and X. YanHong, "Glucagon-like peptide-1 improves proliferation and differentiation of endothelial progenitor cells via upregulating VEGF generation," Medical Science Monitor, vol. 17, no. 2, pp. BR35-BR41, 2011.

[88] W. Wang, J. K. Lang, G. Suzuki, J. M. Canty Jr., and T. Cimato, "Statins enhance clonal growth of late outgrowth endothelial progenitors and increase myocardial capillary density in the chronically ischemic heart," PLoS ONE, vol. 6, no. 9, Article ID e24868, 2011.

[89] C.-H. Wang, I.-C. Hsieh, J.-H. S. Pang et al., "Factors associated with purity, biological function, and activation potential of endothelial colony-forming cells," American Journal of Physiology-Regulatory Integrative and Comparative Physiology, vol. 300, no. 3, pp. R586-R594, 2011.

[90] P. Ji, S. Manupipatpong, N. Xie, and Y. Li, "Induced pluripotent stem cells: generation strategy and epigenetic mystery behind 
reprogramming," Stem Cells International, vol. 2016, Article ID 8415010, 11 pages, 2016.

[91] N. Quiskamp, J. E. Bruin, and T. J. Kieffer, "Differentiation of human pluripotent stem cells into $\beta$-cells: potential and challenges," Best Practice \& Research: Clinical Endocrinology \& Metabolism, vol. 29, no. 6, pp. 833-847, 2015.

[92] X. Y. Chan, R. Black, K. Dickerman et al., "Three-dimensional vascular network assembly from diabetic patient-derived induced pluripotent stem cells," Arteriosclerosis, Thrombosis, and Vascular Biology, vol. 35, no. 12, pp. 2677-2685, 2015. 

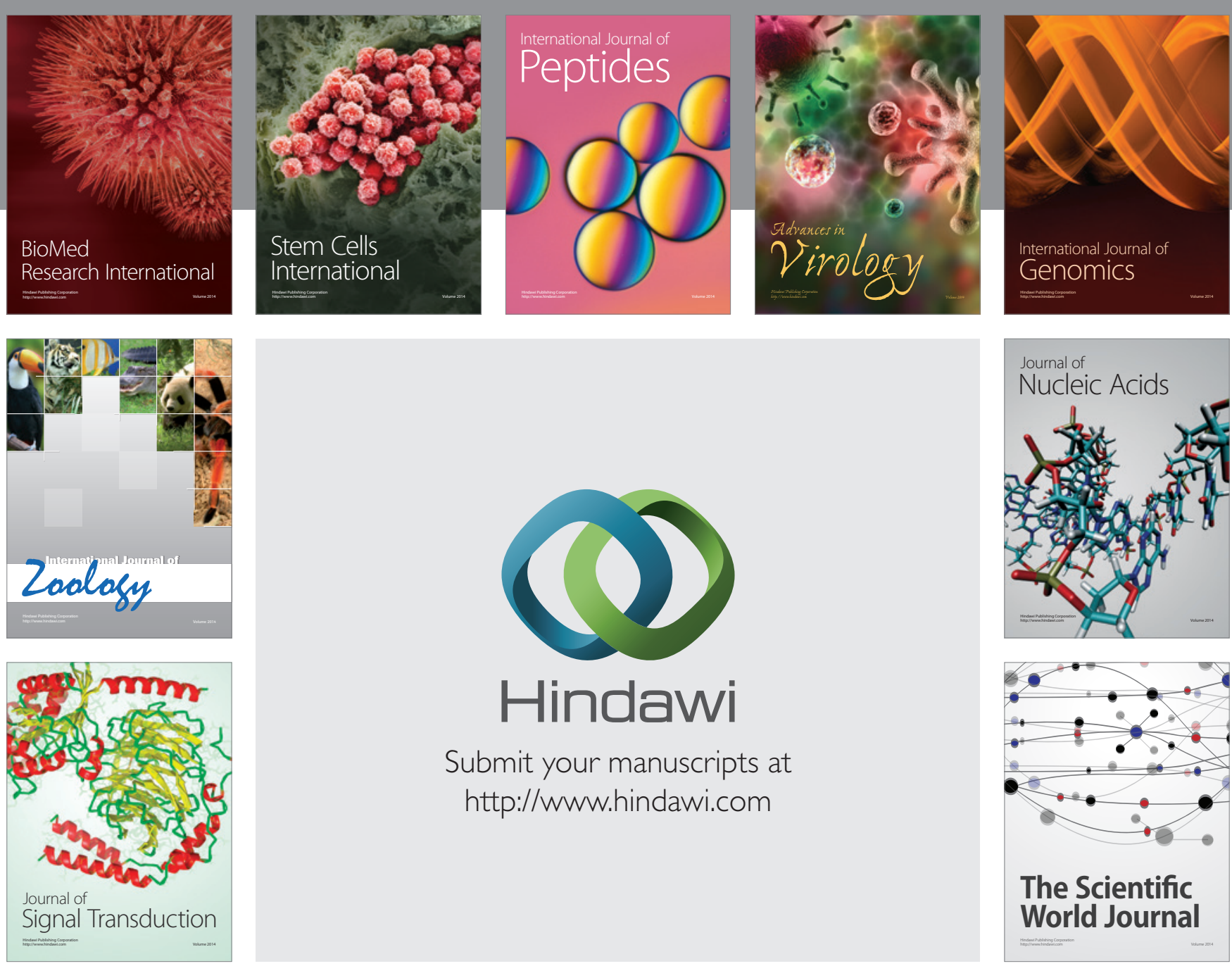

Submit your manuscripts at

http://www.hindawi.com
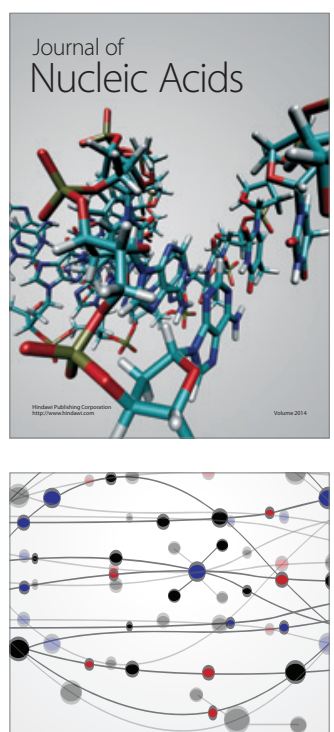

The Scientific World Journal
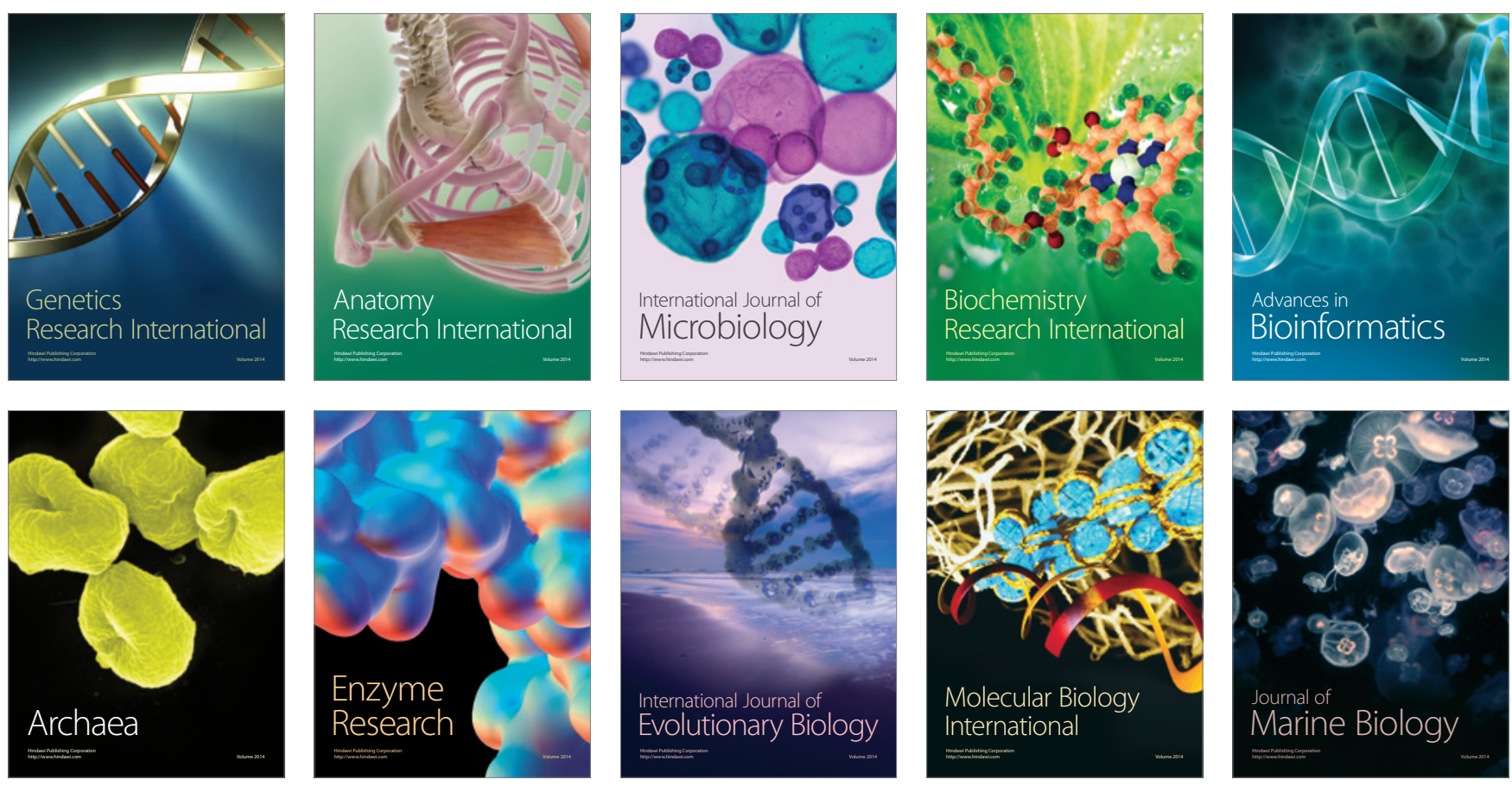